\title{
Racial and Socioeconomic Inequity in the Spatial Distribution of LGBTQ Human Services: an Exploratory Analysis of LGBTQ Services in Chicago
}

Kris Rosentel, Alicia VandeVusse, Brandon J. Hill

\begin{abstract}
LGBTQ people of color and low-socioeconomic status face a heavy burden of health, economic, and social disparities. Understanding the factors that hinder or facilitate efforts to address these disparities is critical to achieving equity and improving social welfare. This study explores one potential factor: the spatial distribution of LGBTQ human services. A spatially referenced dataset of 193 LGBTQ human service sites in the Chicago area was compiled between November 2015 and January 2016. Sites were geocoded and mapped in ArcGIS along with data from the 2011-2015 American Community Survey. Geospatial techniques were used to assess racial and socioeconomic patterns in service distribution. Analyses revealed that sites were disproportionately located in majority non-Hispanic/Latino White (71.0\%) and upper-income (63.7\%) block groups. Additionally, analyses revealed that Black/African American, Hispanic/Latino, and poor to low-income residents of Chicago disproportionately live in LGBTQ service deserts. These patterns in the distribution of LGBTQ services may present barriers to addressing disparities by making services less accessible for racial/ethnic minorities and lower-income individuals. System- and structural-level interventions are needed to reduce spatial inequities in the distribution of LGBTQ human services in Chicago in order to facilitate efforts to address LGBTQ disparities among racial/ethnic minorities and individuals with lower socioeconomic status.
\end{abstract}

Read full article here (no subscription required): https://rdcu.be/bjKxn 